\title{
Perfil epidemiológico dos portadores de helicobacter pylori de acordo com sistema do grupo sanguíneo $A B O$
}

A infecção pelo Helicobacter pylori (HP) depende de fatores genéticos, socioambientais e associa-se patologias gástricas e grupos do sistema ABO. Correlacionar o perfil epidemiológico de portadores de HP com o sistema ABO. Estudo prospectivo, realizado em um serviço de endoscopia digestiva, de abril/2018 a maio/2019. Realizou-se biopsia e histopatologia de mucosa gástrica, teste rápido da uréase e tipagem sanguínea. Aplicado questionário sociodemográfico e clínico. Amostra composta de 44 portadores de HP. Dados analisados pelo R Core team 2019 e submetidos a análises descritivas e inferenciais. Nível de significância 5\%. Todos assinaram o TCLE. Como resultado, $22(50 \%)$ eram mulheres e 22(50\%) homens; média de 55,2 anos (DP=16,7), não havendo significância estatística quando comparado entre os fenótipos do sistema $\mathrm{ABO}$. O fenótipo $\mathrm{O}$ foi prevalente tanto em relação à moradia, profissão e os diversos graus de instrução. Maior frequência do fenótipo $O$ entre os portadores de cor parda e naqueles com infecção pelo HP, sendo 6 (23,1\%) grau de intensidade leve, 10(38,5\%) grau moderado e 10(38,5\%) grau intenso. Diversos fatores contribuem para a transmissão e patogenicidade do HP. Fatores ligados aos genes do indivíduo parecem demonstrar correlação do sistema ABO com doenças gástricas, tendo o fenótipo A maior expressão do LEB, antígeno expresso na mucosa gástrica. Considerando que nem todas as cepas são igualmente específicas para $\mathrm{O}$ e Leb, é possível que diferenças no perfil sociodemográfico e fatores de risco para a contaminação com cepas mais virulentas, possam favorecer a maior suscetibilidade a doenças gástricas

Palavras-chave: Epidemiologia; Fatores de risco; Helicobacter pylori; Sistema do grupo sanguíneo ABO.

\section{Epidemiological profile of helicobacter pylori carriers according to the ABO blood group system}

\begin{abstract}
Helicobacter pylori (HP) infection depends on genetic, socioenvironmental factors and is associated with gastric pathologies and ABO system groups. Correlate the epidemiological profile of patients with $\mathrm{PH}$ with the ABO system. Prospective study, performed in a digestive endoscopy service, from April/2018 to May/2019. Biopsy and histopathology of gastric mucosa, rapid urea test and blood typing were performed. Sociodemographic and clinical questionnaire applied. Sample made up of $44 \mathrm{HP}$ patients. Data analyzed by the R Core team 2019 and submitted to descriptive and inferential analyzes. Significance level $5 \%$. Everyone signed the IC. As a result, $22(50 \%)$ were women and $22(50 \%)$ men; average of 55.2 years ( $S D=16.7)$, with no statistical significance when compared between the $A B O$ system phenotypes. The $\mathrm{O}$ phenotype was prevalent both in relation to housing, profession and varying degrees of education. Higher frequency of phenotype $\mathrm{O}$ among patients with brown skin color and those with HP infection, with 6 (23.1\%) mild degree, 10 (38.5\%) moderate degree and 10 (38.5\%) intense degree. Several factors contribute to the transmission and pathogenicity of $\mathrm{PH}$. Factors linked to the individual's genes seem to demonstrate a correlation of the $\mathrm{ABO}$ system with gastric diseases, with the phenotype The greatest expression of LEB, an antigen expressed in the gastric mucosa. Considering that not all strains are equally specific for $O$ and Leb, it is possible that differences in the sociodemographic profile and risk factors for contamination with more virulent strains, may favor greater susceptibility to gastric diseases.
\end{abstract}

Keywords: Epidemiology; Risk factors; Helicobacter pylori; ABO blood group system.

Topic: Clínica Médica

Reviewed anonymously in the process of blind peer.
Received: $\mathbf{2 1 / 0 2 / 2 0 2 0}$

Approved: 02/05/2020
Durval José de Santana Neto ib

Universidade Tiradentes, Brasil

http://lattes.cnpq.br/8351802057607464

http://orcid.org/0000-0002-5730-5118

durvalneto777@gmail.com

Larissa Gonçalves Moreira (iD)

Universidade Tiradentes, Brasil

http://lattes.cnpq.br/4801033855882414

http://orcid.org/0000-0002-5968-8634

larigoncalvesm@gmail.com

Vinícius Leite de Castro

Universidade Tiradentes, Brasil

http://lattes.cnpq.br/9955888402419446

http://orcid.org/0000-0003-3001-8554

viniciuslcastro@yahoo.com.br

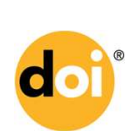

DOI: 10.6008/CBPC2236-9600.2020.002.0006

\author{
Adriana de Oliveira Guimarães (iD \\ Universidade Tiradentes, Brasil \\ http://lattes.cnpq.br/5198069378130459 \\ http://orcid.org/0000-0002-7450-6325 \\ drianabiomedica@hotmail.com \\ Íkaro Daniel de Carvalho Barreto (iD) \\ Universidade Federal Rural de Pernambuco, Brasil \\ http://lattes.cnpq.br/6898002468389405 \\ http://orcid.org/0000-0001-7253-806X \\ daniel.carvalho.ib@gmail.com \\ Leda Maria Delmondes Freitas Trindade (it) \\ Universidade Tiradentes, Brasil \\ http://lattes.cnpq.br/1164446592155027 \\ http://orcid.org/0000-0003-4300-4274 \\ ledeltrin@gmail.com
}

Referencing this:

SANTANA NETO, D. J.; MOREIRA, L. G.; CASTRO, V. L.; GUIMARÃES, A. O.; BARRETO, Í. D. C.; TRINDADE, L. M. D. F.. Perfil epidemiológico dos portadores de helicobacter pylori de acordo com sistema do grupo sanguíneo ABO. Scire Salutis, v.10, n.2, p.43-49, 2020. DOI: http://doi.org/10.6008/CBPC2236-9600.2020.002.0006 


\section{INTRODUÇÃO}

A infecção por Helicobacter pylori (HP) representa uma infecção bacteriana comum em humanos. Ela acomete cerca de $50 \%$ da população mundial e sua descoberta data desde a década de 80 por Marshall e Warren (KODAIRA et al., 2002). A infecção está associada a doenças infecciosas gastrointestinais sejam inflamatórias (gastrite crônica e doença ulcerosa péptica) ou neoplásicas (adenocarcinoma e linfoma MALT) (CORREA et al., 2008; MÉGRAUD, 1995). As vias oral-oral e fecal-oral são consideradas como as principais vias de transmissão da bactéria (NUNEZ et al., 2010).

A infecção pela bactéria na população infantil em países em desenvolvimento pode alcançar 4,0\% a 5,0\% ao ano. Enquanto, nos países desenvolvidos, é de $1 \%$ ao ano em adultos e 2 a $3 \%$ nas crianças e jovens. A prevalência varia de países, em decorrência das diferentes condições socioeconômicas (PARENTE et al., 2010). Até pouco mais de uma década a etiologia de alterações patológicas gástricas eram atribuídas somente ao desequilíbrio entre mecanismos de defesa do hospedeiro e secreção ácida. Estudos associam a presença da bactéria HP e patologias gástricas (DUELL et al., 2015; MEHMET et al., 2005; TEIXEIRA et al., 2016).

A prevalência por HP é variável e depende de fatores como sexo, idade, etnia, dieta, tabagismo, consumo de álcool, alimentos contaminados, consumo de água não tratada, tamanho da família, compartilhamento de cama, más condições de vida na infância, nível educacional e socioeconômico, higiene e histórico médico familiar de doença gástrica (BORGES et al., 2019). Estudos veem demonstrando a interrelação entre as precárias condições de vida das populações brasileiras de baixa renda e a aquisição da infecção por HP. A melhoria do padrão de vida dessas pessoas tem correlação direta com o declínio da infecção humana pelo HP (BORGES et al., 2019; MACHIDA-MONTANI et al., 2004; PARENT, 2010).

No Japão, a partir de 1950, houve considerável declínio da infecção do HP se associando ao desenvolvimento econômico do pós-guerra e à melhoria no saneamento básico (MACHIDA-MONTANI et al., 2004). O sexo masculino foi considerado fator protetor para a infecção pelo HP (ILVER et al., 1998) e a sua prevalência entre homens e mulheres, foi igual em ambos os sexos (GUIMARÃES et al., 2008). Os residentes em zona rural são mais acometidos pela infecção quando comparados com aqueles que residem em zona urbana (SEYDA et al., 2007).

O sistema de grupos sanguíneos $A B O$ é o mais pesquisado sistema antigênico eritrocitário. Esse sistema funciona como marcador genético em estudos de associações com doenças (ALVES et al., 2003). Em 1993, Boren e outros autores, relataram que indivíduos do grupo O seriam mais suscetíveis a contrair HP, pela maior facilidade de adesão, dessa bactéria, ao epitélio gástrico. Indivíduos $\mathrm{O}$ positivo não expressa os antígenos A e B, entretanto apresentam mais receptores para a bactéria $\mathrm{HP}$, quando comparados aos outros grupos do sistema ABO (ILVER et al., 1998). Descobertas relataram que cepas do HP diferem 1500 vezes em relação às afinidades de ligação (KERAMATI et al., 2012).

Atualmente, grandes desafios que envolvem estudos científicos buscam um melhor entendimento da epidemiologia do HP, o que permitirá o desenvolvimento de medidas de prevenção. Este estudo teve 
como proposta correlacionar o perfil epidemiológico de pacientes portadores de HP e o sistema de grupo sanguíneo $A B O$.

\section{METODOLOGIA}

Estudo prospectivo e descritivo, com abordagem analítica quantitativa. Os dados foram coletados durante o período de abril de 2018 a maio de 2019 em um Serviço de Endoscopia Digestiva e Biliopancreática, tendo o Laboratório de Patologia e o Laboratório de Biomedicina do município de Aracaju (SE) como colaboradores na realização dos exames histopatológico e laboratoriais. Foram incluídos pacientes portadores de Helicobacter pylori de ambos os sexos a partir de 17 anos de idade que foram encaminhados para realização de endoscopia digestiva alta (EDA). Foram excluídos portadores de comorbidades descompensadas e indivíduos que não possuíam condições cognitivas para compreender o procedimento e responder o instrumento de pesquisa, com o objetivo de coletar informações quanto ao perfil sociodemográfico e clínico do participante do estudo.

Antes da realização da EDA foi coletada amostra sanguínea, a fim de obter a tipagem do grupo sanguíneo ABO. Todos realizaram preparo adequado conforme protocolo do serviço, sendo devidamente monitorizados quanto a saturação de oxigênio e a frequência cardíaca. Aplicado anestésico tópico na faringe com cloridrato de lidocaína spray a $10 \%$. Para a sedação endovenosa se utilizou fentanil, midazolam e propofol, administrados de forma progressiva até atingir nível adequado de sedação.

Foram coletados amostra de mucosa gástrica através de biopsia para realizar exame histopatológico e teste rápido da urease. Fragmento da mucosa gástrica, foi imerso em meio contendo ureia e indicador de pH. Para o resultado histológico foram obtidos três fragmentos da região de corpo, fundo e antro gástrico, sendo encaminhados para o Laboratório de Patologia.

Para a análise dos dados foram realizadas análises descritivas (frequência simples e percentual), média e desvio padrão e média e análise inferencial, utilizando-se os testes de Qui-Quadrado de Pearson para avaliar a independência de variáveis qualitativas e teste de Mann-Whitney para cálculo das diferenças de medida central. Os dados foram analisados pelo R Core Team 2019, e adotado nível de significância 5\%. Todos assinaram o TCLE (Termo de Consentimento Livre e Esclarecido). Estudo aprovado pelo Comitê de Ética em Pesquisa com Seres Humanos da Universidade Tiradentes-CEP/UNIT sob parecer no 2.522.849 (CAAE: 83071818.5.0000.5371).

\section{RESULTADOS}

A faixa etária da amostra variou de 17 a 81 anos de idade, obtendo-se uma média de 55,2 anos e um desvio padrão (DP) de 16,7. Do total da amostra, os portadores do grupo $O$ apresentaram idade média de $55,6(D P=17,5)$, do grupo $B 55,1(D P=14,8)$, grupo $A B 49,5(D P=23,3$ ) e o grupo $A 55,6(D P=17,3)$, não havendo significância estatística $(p=0,580)$ (Gráfico 1).

Em relação ao total de pacientes infectados por HP, o grupo A representou 20,5\% dos indivíduos; o grupo AB 4,6\%; o grupo B 15,9\% e o grupo O 59\%. Quanto ao grau de intensidade da infecção pelo HP, 14 
pacientes que apresentaram intensidade de infecção moderada $10(38,5 \%)$ e a severa $10(38,5 \%)$ pertenciam ao tipo sanguíneo O. Apenas 1 (14,3\%) representou o tipo B e 3 (33,3 \%) o tipo sanguíneo A (Tabela 1).

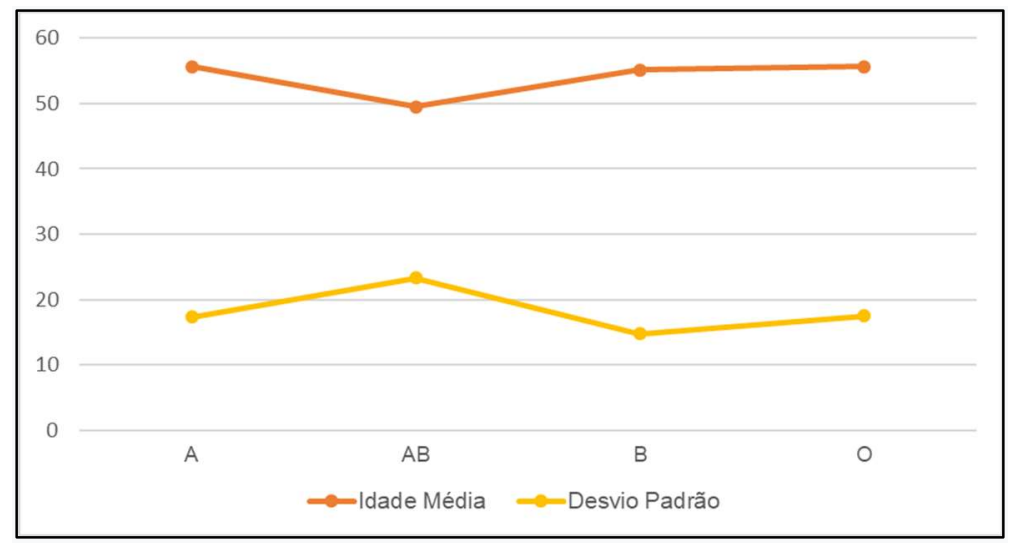

Gráfico 1: Idade Média de portadores de H.pylori submetidos a endoscopia digestiva alta.

Tabela 1: Graus de intensidade da infecção pela H.pylori de acordo com o grupo sanguíneo ABO.

\begin{tabular}{|l|l|l|l|l|l|l|}
\hline & $\mathrm{A}$ & $\mathrm{AB}$ & $\mathrm{B}$ & $\mathrm{O}$ & Total & p-valor \\
\hline H.pylori, $n(\%)$ & & & & & & \\
\hline Leve & $4(44,4)$ & $2(100)$ & $3(42,9)$ & $6(23,1)$ & $15(34,1)$ & 0,363 \\
\hline Moderado & $3(33,3)$ & $0(0,0)$ & $1(14,3)$ & $10(38,5)$ & $14(31,8)$ & \\
\hline Severo & $2(22,2)$ & $0(0,0)$ & $3(42,9)$ & $10(38,5)$ & $15(34,1)$ & \\
\hline
\end{tabular}

Legenda: $n$ - frequência absoluta. \% - frequência relativa percentual. DP - Desvio padrão. ${ }^{\text {QM }}$

Teste Qui-Quadrado de Pearson com simulações de Monte-Carlo. ${ }^{\text {W }}$ Teste de Mann-Whitney.

Daqueles que residiam na capital, $50 \%$ eram do grupo $0,100 \%$ do grupo B, $44,4 \%$ do tipo $A$ e $50 \%$ dos indivíduos $A B$. O tipo sanguíneo $O(40,5 \%)$ foi mais frequente entre aqueles que residiam no interior do estado. O sexo feminino foi mais prevalente no grupo sanguíneo do tipo A 55,6\%, não havendo diferença significativa em relação ao sexo nos grupos $O$ e $A B$. Os homens identificados como sendo grupo $B$, foram mais acometidos pela bactéria HP $(57,1 \%)$. Quanto à cor de pele, a parda $(56,5 \%)$ foi mais prevalente entre os portadores do fenótipo $\mathrm{O}$ (Tabela 2).

Tabela 2: Perfil sociodemográfico de portadores de $H$. pylori submetidos a endoscopia digestiva alta.

\begin{tabular}{|c|c|c|c|c|c|c|}
\hline H. pylori positivo & A & $A B$ & $\mathrm{~B}$ & 0 & Total & $\mathrm{p}$-valor \\
\hline Idade, Média (DP) & $55,6(17,3)$ & $49,5(23,3)$ & $55,1(14,8)$ & $55,6(17,5)$ & $55,2(16,7)$ & $0,580 \mathrm{w}$ \\
\hline \multicolumn{7}{|l|}{ Zona, $n(\%)$} \\
\hline -Capital & $4(44,4)$ & $1(50)$ & $7(100)$ & $12(50)$ & $24(57,1)$ & $0,282 \mathrm{QM}$ \\
\hline -Interior & $5(55,6)$ & $1(50)$ & $0(0)$ & $11(45,8)$ & $17(40,5)$ & \\
\hline -Outros Estados & $0(0)$ & $0(0)$ & $0(0)$ & $1(4,2)$ & $1(2,4)$ & \\
\hline \multicolumn{7}{|l|}{ Sexo, $n(\%)$} \\
\hline -Feminino & $5(55,6)$ & $1(50)$ & $3(42,9)$ & $13(50)$ & $22(50)$ & $1,000 \mathrm{QM}$ \\
\hline -Masculino & $4(44,4)$ & $1(50)$ & $4(57,1)$ & $13(50)$ & $22(50)$ & \\
\hline \multicolumn{7}{|l|}{ Profissão, $n$ (\%) } \\
\hline -Empregado com carteira assinada & $0(0)$ & $0(0)$ & $0(0)$ & $3(11,5)$ & $3(6,8)$ & $0,306^{Q M}$ \\
\hline -Empregado sem carteira assinada & $3(33,3)$ & $1(50)$ & $1(14,3)$ & $4(15,4)$ & $9(20,5)$ & \\
\hline -Desempregado & $0(0)$ & $1(50)$ & $1(14,3)$ & $2(7,7)$ & $4(9,1)$ & \\
\hline -Aposentado & $6(66,7)$ & $0(0)$ & $3(42,9)$ & $7(26,9)$ & $16(36,4)$ & \\
\hline -Estudante & $0(0)$ & $0(0)$ & $0(0)$ & $2(7,7)$ & $2(4,5)$ & \\
\hline -Autônomos & $0(0)$ & $0(0)$ & $2(28,6)$ & $8(30,8)$ & $10(22,7)$ & \\
\hline \multicolumn{7}{|l|}{ Grau de instrução, $n$ (\%) } \\
\hline -Analfabeto & $2(22,2)$ & $1(50)$ & $1(20)$ & $6(26,1)$ & $10(25,6)$ & 0,418 QM \\
\hline -Ensino Fundamental & $2(22,2)$ & $0(0)$ & $4(80)$ & $5(21,7)$ & $11(28,2)$ & \\
\hline -Ensino Médio & $4(44,4)$ & $1(50)$ & $0(0)$ & $9(39,1)$ & $14(35,9)$ & \\
\hline -Ensino Superior & $1(11,1)$ & $0(0)$ & $0(0)$ & $3(13)$ & $4(10,3)$ & \\
\hline Cor da Pele, $n(\%)$ & & & & & & \\
\hline
\end{tabular}




\begin{tabular}{|l|l|l|l|l|l|l|}
\hline -Branco & $2(22,2)$ & $1(50)$ & $2(40)$ & $3(13)$ & $8(20,5)$ & 0,804 QM \\
\hline -Negros & $2(22,2)$ & $0(0)$ & $1(20)$ & $7(30,4)$ & $10(25,6)$ & \\
\hline -Pardo & $5(55,6)$ & $1(50)$ & $2(40)$ & $13(56,5)$ & $21(53,8)$ & \\
\hline -Amarelo & $4(44,4)$ & $1(50)$ & $7(100)$ & $12(50)$ & $24(57,1)$ & \\
\hline
\end{tabular}

Legenda: $\mathrm{n}$ - frequência absoluta. \% - frequência relativa percentual. DP - Desvio padrão. QM Teste Qui-Quadrado de Pearson com simulações de Monte-Carlo. ${ }^{\mathrm{W}}$ Teste de Mann-Whitney.

\section{DISCUSSÃO}

A prevalência da infecção por Helicobacter pylori varia com o sexo, idade, nível socioeconômico e cor de pele. Em nosso estudo foi encontrado idade média de 55,2 anos, sendo compatível com outros relatos em que, a prevalência da infecção por HP, aumenta com a idade e é maior nos países em desenvolvimento (LADEIRA et al., 2003).

A soroprevalência do HP é igual em homens e mulheres (GO, 2002; KERAMATI et al., 2012). Observouse divergência entre alguns estudos, no que se refere a prevalência de infecção por HP, quanto ao sexo (GO, 2002; TADESSE et al., 2014). No presente estudo, os dados se revelaram diferentes, visto que, não houve significancia estatística entre os sexos. Autores afirmam maior taxa de infecção por HP entre aqueles que residem em zonas rurais em comparação com a zona urbana (TADESSE et al., 2014). Esse fato difere dos resultados encontrados neste estudo, visto que, a maior taxa encontrada foi de residentes da capital do estado. A infecção pelo H.pylori tem sido mais comum em população não branca (KODAIRA et al., 2002). Dentre todas as cores de pele identificadas no estudo, a parda foi a mais prevalente $(57,1 \%)$.

A aglomeração intrafamiliar é um fator importante para infecção do HP. Existe uma compatibilidade de sorologia positiva para HP em mais de $80 \%$ entre irmãos conviventes no mesmo lar, havendo maior incidência do HP em crianças que possuem pais infectados (LADEIRA et al., 2003). É possível que índices menores de baixa escolaridade aproxima a correlação entre baixo desenvolvimento socioeconômico e a infecção pela bactéria. O estudo identificou quatro (10,3 \%) indivíduos com ensino superior e dez (25,6 \%) analfabetos, baixo índice de emprego com mão de obra qualificada, e apenas três (11,5 \%) tinham empregos com carteira assinada. Tal evidência pode ser atribuída a baixa condição socioeconômica, o que propicia moradias em áreas de saneamento básico precário, tornando-se um fator de risco para a contaminação por HP (TADESSE et al., 2014).

Além das causas ambientais, os fatores genéticos também contribuem na transmissão e patogenicidade do H. pylori (KODAIRA et al., 2002). Em relação aos fatores ligados aos genes do indivíduo, observa-se a correlação do sistema de grupos sanguíneos ABO com doenças gástricas (BARRETO et al., 2014). Assim, tal sistema antígeno eritrocitário tem sido estudado na tentativa de melhor compreender como alguns indivíduos têm predisposição a se infectar pela bactéria, e o porquê de outros, depois de infectados não desenvolverem alterações patológicas gástricas. 0 entendimento deste questionamento justifica-se pela secreção dos antígenos de Lewis que são bioquimicamente relacionados aos aloantígenos do sistema $A B O$. Assim, os indivíduos podem ou não secretarem antígenos de Lewis $\mathrm{ABH}$.

A correlação com a bactéria $H$. pylori diz respeito ao fato de que, a bactéria adere aos receptores de superfície celular através da produção de adesinas BabA e SabA, que também se ligam a antígenos dos grupos 
sanguíneos Lewis a-1 e Lewis B. O grupo O teria maior expressão do Leb, antígeno expresso na mucosa gástrica com maior afinidade receptora para fixação por HP, indicando que, indivíduos do grupo sanguíneo O, seriam mais susceptíveis à infecção pelo H. pylori (BORGES et al., 2019; DUELL et al., 2015; MC et al., 2017; RIZZATO et al., 2013; TRINDADE et al., 2017).

\section{CONCLUSÕES}

O estudo demonstrou que portadores da bactéria $H$. pylori mantém uma relação entre o sistema $\mathrm{ABO}$, principalmente entre aqueles que apresentavam um grau de infecção moderado a severo. O perfil epidemiológico desses pacientes parece favorecer a uma condição de contaminação principalmente naqueles que apresentaram fenótipo O. Considerando que nem todas as cepas são igualmente específicas para $\mathrm{O}$ e Leb, é possivel que diferenças no perfil sociodemográfico e fatores de risco que favoreçam a contaminação com cepas mais virulentas, possam favorecer a maior suscetibilidade a desenvolverem doenças gástricas. Estudos futuros, envolvendo amostras mais significativas e identificação dos aloantígenos do sistema $A B O$, possibilitarão dados mais evidentes quanto à suscetibilidade à infecção pelo Helicobacter pylori. O conhecimento de sua patogênese e a correlação com dados epidemiológicos, tende a ser um importante mecanismo de prevenção, visto que é considerada um importante problema de saúde pública.

\section{REFERÊNCIAS}

ALVES, R. T.; MATTOS, A. L. C.; FERRARI, F.; BONINIDOMINGOS, C. R.. Avaliação do polimorfismo de grupos sanguíneos e fenótipo de hemoglobinas em um grupo de universitários de São José do Rio Preto, SP. Rev. Bras. Hematol. Hemoter, v.25, n.1, p.65-71, 2003.

BARRETO, M. C.; NUNES, M. P.; PEREIRA, W. S.; BORGES, L. L.; PENNA, K. G. B. D.. Correlação entre o sistema abo e doenças gastrointestinais: revisão sistemática. Revista EVS, Goiânia, v.41, n.4, p.777-784, 2014.

BORGES, S. S.; RAMOS, A. F. P. L.; MORAES FILHO, A. V.; BRAGA, C. A. S. B.; CARNEIRO, L. C.; BARBOSA, M. S.. Prevalência da infecção por Helicobacter pylori em pacientes dispépticos e associação com fatores de riscos clínicos para o desenvolvimento de adenocarcinoma gástrico. Arq. Gastroenterol, 2019.

CORREA, P.; PIAZUELO, M. B.. Natural history of Helicobacter pylori infection. Digestive Liver Disease, v.40, p.490-496, 2006.

DUELL, E. J.; BONET. C.; MUÑOZ, X.; LUJAN-BARROSO, L.; WEIDERPASS, E.; BOUTRON-RAUALT, M. C.; RACINE, A.; SEVERI, G.; CANZIAN, F.; RIZZATO, C.; BOEING, H.; OVERVAD, K.; TJØNNELAND, A.; ARGÜELLES, M.; SÁNCHEZ-CANTALEJO, E.; CHAMOSA, S.; HUERTA, J. M.; BARRICARTE, A.; KHAW, K. T.; WAREHAM, N.; TRAVIS, R. C.; TRICHOPOULOU, A.; TRICHOPOULOS, D.; YIANNAKOURIS, N.; PALLI, D.; AGNOLI, C.; TUMINO, R.; NACCARATI, A.; PANICO, S.; MESQUITA, H. B. B.; SIERSEMA, P. D.; PEETERS, P. H.; OHLSSON, B.; LINDKVIST, B.; JOHANSSON, I.; YE, W.; JOHANSSON, M.; FENGER, C.; RIBOLI, E.; SALA, N.; GONZÁLEZ, C. A.. Variationat ABO histoblood group and FUT loci and diffuseand intestinal gastric cancerrisk in a European population. Int. J. Cancer, v.136, p.880-893, 2015

GO, M. F.. Review article: natural history and epidemiology of Helicobacter pylori infection. Aliment Pharmacol Ther, v.16, n.1, p.3-15, 2002.

GUIMARÃES, J.; CORVELO, T. C.; BARILE, K. A.. Helicobacter pylori: fatores relacionados à sua patogênese. Revista Paraense de Medicina, v.22, n.1, 2008.

ILVER, D.; ARNQVIST, A.; KERSULYTE, D. F.; INCECIK, E. T.; BERG, D. E.. Helicobacter pylori adhes in binding fucosy lated histo-blood group antigens revealed by retagging. Science, v.279, n.5349, p.373-377, 1998.

KERAMATI, M. R.; SADEGHIAN, M. H.; AVYATOLLAHI, H.; BADIEE, Z.; SHAKIBAYI, H.; MOGHIMI-ROUDI, A.. Role of the Lewis and $\mathrm{ABO}$ Blood Group Antigens in Helicobacter pylorilnfection. Malays J. Med. Sci., v.19, n.3, p.17-21, 2012.

KODAIRA, S. M.; ESCOBAR, A. M. U.; GRISI, S.. Aspectos epidemiológicos do Helicobacter pylori na infância e adolescência. Rev. Saúde Pública, v.36, n.3, p.356-69, 2006.

LADEIRA, M. S. P.; SALVADORI, D. M. F.; RODRIGUES, M. A. M.. Biopatologia do Helicobacter pylori. Jornal Brasileiro de Patologia e Medicina Laboratorial, v.39, n.4, p.335-342, 2003.

MACHIDA-MONTANI, A.; SASAZUKI, S.; INOUE, M.; NATSUKAWA, S.; SHAURA, K.; KOIZUMI, Y.; KASUGA, Y.; HANAOKA, T.; TSUGANE, S.. Association of Helicobacter pylori infection na denvironment al factors in non- 
cardiagastriccancer in Japan. Gastric Cancer, v.7, n.1, p.4656, 2004.

MÉGRAUD, F.. Epidemiology of Helicobacter pylori infection: where are in 1995. Eur. J. Gastroenterol Hepatol, v.7, p.292295, 1995.

MEHMET, K.; GUR, G.; ARSLAN, H.; YILMAZ, U.; BOYACIO, S. . The Relationship of ABO Blood Group, Age, Gender, Smoking, and Helicobacter pylori Infection. Digestive Diseases and Sciences, v.50, n.7, 2005.

NUNES, M. R. Z.; MEDEIROS, M. S.; DURLI, M. A.; GUARESI, J. R.; MEDEIROS, V. S.; SAKAE, T. M.. Avaliação das indicações para pesquisa diagnóstica do Helicobacter pylori em serviço ambulatorial de Endoscopia Digestiva. Arquivos

Catarinenses de Medicin., v.39, n.3, 2010.

PARENTE, J. M. L.; PARENTE, M. P. P. D.. Contexto epidemiológico atual da infecção por Helicobacter pylori. GED Gastroenterol. Endosc. Dig., v.29, n.3, p.86-89, 2010.

RIZZATO, C.; KATO, I.; PLUMMER, M.; MUNOZ, N.; STEIN, A.; DOORN, L. I. V.; FRANCESCHI, S.; CANZIAN, F.. Cancer: Risk of advanced gastric precancerous lesions in Helicobacter pylori infected subjects isinfluenced by $\mathrm{ABO}$ blood group and cagA status. Int. J. Cancer, v.133, n.2, p.315-323, 2013.

SEYDA, T.; DERYA, C.; FUSUN, A.; MELIHA, K.. The relationship of Helicobacter pylori positivy with age, sex, and $\mathrm{ABO} /$ Rhesus blood groups in patients with gastrointestinal complaints in Turkey. Helicobacter, v.12, n.3, p.224-250, 2007.

TADESSE, E.; DAKA, D.; YEMANE, D.; SHIMELIS, T. Seroprevalence of Helicobacter pylori infection and its related risk factors in symptomatic patients in southern Ethiopia. BMC Res Notes, v.24, n.7, p.834, 2014.

TEIXEIRA, T. F.; SOUZA, I. K. F.; ROCHA, R. D. R.. Helicobacter pylori infecção, diagnostico laboratorial e tratamento. Percurso Acadêmico, Belo Horizonte, v.6, n.12, 2016.

TRINDADE, L. M. D. F.; MENEZES, L. B.; O.; SOUZA NETA, M. A.; ROLEMBERG, P. C. L.; SOUZA, L. D.; BARRETO, I. D. C.; MEURER, L.. Prevalence of helicobacter pylori in inection of gastric specimens. Prevalence of helicobacter pylori infection. Gastroenterology Research, v.10, n.1, p.33-41, 2017.

A CBPC - Companhia Brasileira de Produção Científica (CNPJ: 11.221.422/0001-03) detém os direitos materiais desta publicação. Os direitos referem-se à publicação do trabalho em qualquer parte do mundo, incluindo os direitos às renovações, expansões e disseminações da contribuiç̃o, bem como outros direitos subsidiários. Todos os trabalhos publicados eletronicamente poderão posteriormente ser publicados em coletâneas impressas sob coordenação da Sustenere Publishing, da Companhia Brasileira de Produção Científica e seus parceiros autorizados. Os (as) autores (as) preservam os direitos autorais, mas não têm permissão para a publicação da contribuição em outro meio, impresso ou digital, em português ou em tradução. 